\section{Pratiques dialogiques et expériences formatives au service du développement professionnel continu des enseignants}

Práticas dialógicas e experiências formativas a serviço do desenvolvimento profissional contínuo dos professores

Dialogical practices and formative experiences for teachers' continuing professional development

\section{Fabienne Brière (D)}

fabienne.briere@univ-amu.fr

INSPÉ Aix-Marseille Université

\section{Laurence Espinassy (D)}

laurence.espinassy@univ-amu.fr

INSPÉ Aix-Marseille Université

\section{Résumé}

Cet article s'intéresse à la dimension dialogique instaurée par le cadre d'une intervention-recherche en milieu scolaire en France qui porte sur la mise en œuvre de l'évaluation par compétences au cycle 3 au sein d'un réseau d'éducation prioritaire renforcé. En référence au cadre théorique et méthodologique de l'ergonomie de l'activité des enseignants dans leur milieu de travail, le dispositif repose sur la co-analyse des situations d'évaluation entre enseignants et chercheurs lors d'entretiens d'autoconfrontations simples et croisées puis de séances collectives de travail. L'analyse des résultats montre comment les acteurs, grâce à la dimension dialogique et formative du dispositif de recherche, prennent conscience de certains usages de dispositifs d'évaluation, découvrent ceux des autres et en envisagent de nouveaux. Elle révèle les difficultés, les compromis opératoires et les déplacements qu'ils opèrent pour établir une véritable continuité dans le parcours des élèves malgré des cultures professionnelles différentes et des prescriptions parfois dissonantes entre quatre écoles et un collège. Les rapports langagiers des enseignants à leur milieu de travail permettent aux chercheurs d'identifier des indices du développement du pouvoir d'agir des acteurs.

Mots-clés

Activité enseignante. Dialogisme. Évaluation par compétences. Formation des enseignants. Intervention-recherche.

\section{Resumo}

Este artigo volta-se para a dimensão dialógica instaurada por uma intervenção-pesquisa em meio escolar na França que trata da implementação da avaliação por competências no ciclo 3 no seio de uma rede de educação prioritária reforçada. No que se refere ao quadro teórico e metodológico da ergonomia da atividade docente em seu meio de trabalho, o dispositivo repousa na coanálise de situações de

\section{Linguagem Foco}

Revista do Programa de Pós-Graduação em Linguística Aplicada da UECE

FLUXO DA SUBMISSÃO

Submissão do trabalho: 12/03/2021 Aprovação do trabalho: 22/04/2021 Publicação do trabalho: 04/06/2021

\section{COMO CITAR}

BRIÈRE, Fabienne; ESPINASSY, Laurence. Pratiques dialogiques et expériences formatives au service du développement professionnel continu des enseignants. Revista Linguagem em Foco, v.13, n.1, 2021. p. 44-65. Disponívelem:https://revistas. uece.br/index.php/linguagememfoco/article/view/5482. 
avaliação entre docentes e pesquisadores durante entrevistas de autoconfrontações simples e cruzadas em seguida de sessões coletivas de trabalho. A análise dos resultados mostra como os atores, graças à dimensão dialógica e formativa do dispositivo de pesquisa, tomam consciência de alguns usos de dispositivos de avaliação, descobrem aqueles de outros e vislumbram novos (dispositivos). Ela revela as dificuldades, os compromissos operatórios e os deslocamentos que eles operam para estabelecer uma verdadeira continuidade no percurso dos alunos apesar das diferentes culturas profissionais e das prescrições algumas vezes dissonantes entre escolas e colégio. As relações linguageiras do indivíduo com seu meio de trabalho permite aos pesquisadores identificar indícios do desenvolvimento do poder de agir desses atores.

\title{
Palavras-chave
}

Atividade docente. Dialogismo. Avaliação por competências. Formação de professores. Intervenção-pesquisa.

\begin{abstract}
This article focuses on the dialogical dimension established by the framework of an intervention-research project in the French school system, which concerns the implementation of evaluation by competencies in cycle 3 within a reinforced priority education network. Referring to the theoretical and methodological framework of the ergonomics of teachers' activity in their work environment, the system is based on the co-analysis of evaluation situations between teachers and researchers during simple and cross-confrontation interviews, followed by collective work sessions. The analysis of the results shows how the actors, thanks to the dialogical and formative dimension of the research device, become aware of certain uses of evaluation devices, discover those of others and envisage new ones. It reveals the difficulties, the operational compromises and the shifts they make to establish a real continuity in the students' pathway despite different professional cultures and sometimes dissonant prescriptions between schools and colleges. The language relations of the individual to his work environment allows the researchers to identify the indices of the development of the actors' power to act.
\end{abstract}

Keywords

Teaching activity. Dialogism. Competency-based assessment. Teacher training. Intervention-research.

\section{Introduction}

Considérée comme un enjeu majeur de l'évolution des systèmes éducatifs, la formation des enseignants fait l'objet d'une réflexion accrue dont témoignent les récentes recherches internationales. Dans ce contexte, nombre de réformes s'attachent à repenser la formation initiale autour d'un partenariat resserré entre institutions universitaire et scolaire (HAYMORE SANDHOLTZ, 2002). Dans ces dispositifs en alternance, il s'agit de permettre aux futurs enseignants de circuler entre expériences pratiques, formation théorique à/par la recherche et analyse réflexive afin de favoriser l'acquisition de compétences professionnelles (COFFEY, 2010 ; VEILLARD; COPPE, 2009). De fait, l'alternance « contribue à une réorganisation des curricula et des dispositifs de formation dans et par les situations de travail » (MAUBANT; ROQUET, 2016, p. 2). Elle repose sur une organisation dans le temps et l'espace qui tend à favoriser une réflexion professionnelle collective (GLAZER ; ABBOTT; HARRIS, 2004) alimentée par l'analyse des pratiques effectives en classe des formés accompagnés de tuteurs de terrain et universitaires.

Plus récemment, en lien avec ces évolutions et malgré des divergences dans les politiques éducatives des différents pays, la focale se déplace sur la formation continue des enseignants pensée autour d'un continum tout au long de 
la carrière (MALET et al., 2021) : l'acquisition d'une véritable culture professionnelle relève d'un processus long et continu qui ne saurait se limiter à l'entrée dans le métier. Les enquêtes internationales s'accordent sur l'importance du développement professionnel continu pour l'amélioration et la qualité des systèmes éducatifs (MALET et al., 2021). En effet, les connaissances acquises par les professeurs au cours de la formation (initiale ou continue) influencent les stratégies pédagogiques qu'ils adoptent et la qualité de leur enseignement (BLÖMEKE; GUSTAFSSON; SHAVELSON, 2015). Si le développement professionnel se manifeste par l'acquisition et la « mobilisation créative » en contexte de nouvelles connaissances, compétences et attitudes (MALET, 2021), son efficacité dépend également des processus de formation (LESSARD, 2021). Selon cet auteur, l'efficacité du développement professionnel continu tient entre autres à l'ancrage des contenus de formation dans les pratiques réelles de classe, au caractère actif des apprentissages des enseignants, à la collaboration entre enseignants, à un soutien expert dans le temps ainsi qu'au développement de la réflexivité. Autrement dit, le développement professionnel se caractérise par l'élaboration collective de nouvelles ressources adaptées aux contraintes du réel (WANLIN; CRAHAY, 2012). II s'inscrit dans une analyse réflexive de son activité d'enseignement et difficultés tant subjectives qu'objectives qu'elle génère (AMIGUES, 2009 ; SAUJAT; FÉLIX, 2018) ; le développement du pouvoir d'agir serait rendu possible par la mise en discussion des questions de métier et des dilemmes vécus (CLOT, 2008a). Ces orientations sont largement soutenues par les modalités de formation centrées sur l'analyse de l'activité et adossés au travail réel que nécessite l'exercice du métier (MAYEN, 2008 ; SIMONET, 2019 ; VINATIER, 2009). Elles mettent en avant le rôle essentiel de l'activité langagière et dialogique dans les processus réflexifs-discursifs (BALSLEV; TOMINSKA; VANHULLE, 2011) qui en retour amènent à repenser la place du formateur dans l'accompagnement du développement professionnel (BRIÈRE ; SIMONET, 2021).

Dans la continuité de ces travaux, nous nous intéressons dans cet article à une situation de développement professionnel d'enseignants d'un réseau d'éducation prioritaire renforcé (REP+) dans le cadre d'une intervention-recherche'. S'inscrivant dans une tradition ergonomique, les interventions-recherches reposent sur une co-analyse entre chercheurs et acteurs professionnels des situations de travail selon une visée développementale (CLOT, 2008b ; SAUJAT ; FÉLIX,

1 Réalisée dans le cadre d'un appel à projet académique entre la structure fédérative de recherche en éducation (SFERE-Provence) et la Délégation Académique à la Formation et à I'Innovation Pédagogique du rectorat d'Aix-Marseille (BRIÈRE-GUENOUN ; ESPINASSY, 2019). 
2018; ESPINASSY; BRIĖRE-GUENOUN ; FÉLIX, 2018). Cette recherche, qui répondait à une demande du chef d'établissement du collège, pilote du réseau REP+, avait pour enjeu de comprendre et d'accompagner les enseignants à mettre en place l'évaluation par compétences au sein du cycle $3^{2}$ relatif à la période scolaire de transition entre les premier et second degrés.

\section{Cadre théorique et méthodologique}

L'intervention en milieu de travail a pour objectif de dépasser la contradiction «entre une démarche explicative qui dissout son objet sans retour et une démarche compréhensive qui le rejoint trop vite " (CLOT, 2008b, p. 74). La question du développement de l'expérience individuelle et collective, permis par une activité dialogique, constitue un objet central des interventions-recherches fondées sur l'analyse de l'activité.

\section{7 L'analyse de l'activité source de développement}

Dans la situation vécue, l'activité « est simultanément tournée vers l'objet immédiat de l'action et vers l'activité des autres portant sur ces objets » (CLOT, 2000, p. 135). En référence aux distinctions établies par Leontiev (1984) entre les concepts d'action, d'opération et d'activité, le développement du pouvoir d'agir des professionnels s'enracine dans le sens qu'attribuent ces derniers à leur activité en lien avec l'efficacité et l'efficience de leurs actions. Ainsi, plus les actions se transforment en opérations, plus le sujet peut s'ouvrir de nouvelles perspectives, de nouveaux buts en produisant un changement de sens de la situation vécue. On fait l'hypothèse que les situations créées et leur transformation initient un triple mode de développement : a) des situations examinées, par la remise en travail dont elles font l'objet de la part des acteurs, b) des sujets engagés, par la découverte des dimensions non réalisées ou non perçues de leur activité et la conception des façons de s'en libérer, et c) des collectifs, par la formalisation, la reconnaissance ou le dépassement des modalités communes de l'action.

De façon spécifique, les professeurs organisent leur milieu de travail pour concevoir ce qu'ils ont à faire et savoir comment le faire. L'activité de l'enseignant en classe consiste à organiser un milieu de travail collectif pour instaurer chez les élèves un rapport culturel à un objet de savoir, afin de modifier leur rapport personnel à ce savoir. Elle concerne l'organisation collective du milieu-classe, la distribution des tâches aux élèves dans le temps, l'organisation d'un dialogue di- 
dactique, la construction du sens du faire et du dire ainsi que sa reprise dans I'histoire du groupe classe. Pour autant, l'activité professorale ne se limite pas au seul milieu de la classe mais se situe à l'articulation de plusieurs logiques, plusieurs temporalités mettant en jeu divers partenaires. En effet, elle s'inscrit dans un travail de conception qui relève de dimensions collectives (avec des collègues de travail) plus ou moins visibles dans l'établissement. Dans ce cadre, l'analyse de l'activité s'intéresse à la fois à ce que le professeur fait avec ses élèves dans le but de faire apprendre mais aussi à l'effet du travail sur le professeur lui-même. Parce que l'activité du professeur engage celui-ci dans un ensemble de dilemmes exigeant des compromis, son analyse cherche à éclairer la façon dont les choix réalisés par l'enseignant mettent à l'épreuve les prescriptions institutionnelles, ses convictions professionnelles, ses exigences envers ses élèves ou envers lui-même et l'affirmation de sa position professionnelle auprès de ses collègues. Le développement de l'expérience professionnelle du professeur suppose une mise en dialogue de ce dernier avec son travail et avec ses pairs dans un rapport circulaire entre théorie et pratique. Dans cette optique, l'analyse individuelle et collective de situations concrètes de travail/enseignement-apprentissage peut soutenir l'activité dialogique.

\section{2 Un développement ancré dans un mouvement dialogique}

L'expression de l'expérience professionnelle est tributaire d'une double épreuve : celle d'une mise en travail de la pensée des opérateurs à travers sa mise en mots, d'une part, et celle de la confrontation aux mots des autres avant leur réintégration dans leur potentiel discursif, d'autre part. En effet, « en se transformant en langage la pensée se réorganise et se modifie. Elle ne s'exprime pas mais se réalise dans le mot » (VYGOTSKI, 1997, p. 428).

Selon ce point de vue, considérer l'analyse de l'activité comme moteur du développement nécessite donc d'ouvrir un « espace-temps » (CLOT ; FAÏTA, 2000) où se déploie un rapport dialogique au sens de Bakhtine, c'est-à-dire irréductible à l'enchaînement des répliques entre deux sujets engagés dans une interaction. Ce rapport implique non seulement que le sujet soit confronté à son propre travail mais aussi à celui de ses pairs par le truchement de l'analyse langagière. Ce dialogue qui s'instaure avec son activité et celle de ses pairs au contact des actions professionnelles effectives menées par chacun d'entre eux permet d'ouvrir un champ de possibilités nouvelles à un entrecroisement d'activités elles-mêmes nouvelles, en l'occurrence dialogiques. Autrement dit, cet empilement « d'activi- 
tés sur l'activité » contribue à convertir l'expérience individuelle en une « monnaie collective qui (...) peut entrer en circulation sur une base plus large que la seule relation interpersonnelle »(BRUNER, 2000, p. 114). Finalement, se raconter des histoires, «c'est jeter un pont entre ce qui est établi et ce qui est possible », c'est une "recherche de compromis entre autonomie et engagement alimenté par un processus dialectique entre ce qui nous est familier et ce qui serait possible » (SIMONET, 2005, p. 2).

Ces orientations peuvent alimenter un espace de formation composite plein d'histoires et de situations possibles, de discours entremêlés concernant l'activité de travail des professeurs (ESPINASSY, 2016). Faire dialoguer entre eux les différents acteurs autour des ressemblances et dissemblances des manières de faire leur métier permet, potentiellement, d'ouvrir en chacun une gamme enrichie de possibilités futures qui s'enracinent dans une arène de conflits de critères et de débats professionnels entre gens de métier (WALLON, 1970). Ces conflits de critères à propos des façons de faire et de penser le métier amènent des processus de renormalisation (SCHWARTZ, 1997) 3 à condition que les difficultés vécues par les enseignants sur un mode personnel trouvent l'occasion de se convertir en un questionnement partagé par un collectif de travail. Concrètement, il s'agit d'établir un cadre méthodologique privilégiant la création de conditions propices à l'enchaînement de ce double processus : réalisation de la pensée dans le discours puis confrontation au discours, à la voix d'autrui au sujet de sa propre activité. L'enjeu d'une telle démarche est de permettre aux acteurs de reprendre la main sur des aspects et dimensions de la situation qui leur avaient primitivement échappés en leur donnant l'occasion de revivre leur vécu et de discuter de leur métier grâce à l'instauration de nouveaux contextes.

\section{3 Une méthodologie indirecte au service de la formation des enseignants}

Le développement de l'expérience n'est pas immédiatement accessible par l'observation directe, mais à partir de «traces » qu'il faut reconstruire comme le propose le cadre méthodologique de «l'auto-confrontation » en tant que « méthode indirecte » (VYGOTSKI, 1994). Ancré dans d'une approche historico-culturelle (VYGOTSKI, 1997) et dialogique (CLOT; FAÏTA, 2000), ce cadre offre un milieu propice à la transformation des situations de travail par les professionnels euxmêmes placés en situation d'analyse de leur activité.

Dans une conception Vygotskienne, l'action matérielle reconstruite ver-

3 C'est-à-dire la redéfinition en contexte de ses propres normes. 
balement dans l'interaction sociale sur le plan de la conscience se transforme en une action qui s'organise sur de nouvelles bases. Cette démarche qui repose sur « l'expérience vécue d'une expérience vécue » (VYGOTSKI, 1994, p. 78) entraîne un processus de «traduction d'une activité en une autre activité, [une] liaison entre activités » (CLOT, 2003, p. 12). En référence à l'orientation théorique de l'ergonomie de l'activité selon une approche clinique (FAÏTA; SAUJAT, 2010), le cadre méthodologique de l'autoconfrontation ${ }^{4}$ (CLOT; FAÏTA, 2000) rend possible la transition d'une activité première (vidéoscopée) en une activité à venir par la relation avec une troisième : l'activité dialogique proposée. Selon ce point de vue, les mises en relation entre les énoncés, les actions et les situations peuvent devenir ellesmêmes objet de réflexion et de débat, à travers la reconnaissance de la pluralité des voix et des signes qui composent la dimension concrète des échanges verbaux. Au dialogue contraint, situé, contingent aux modes d'évaluation sociaux et techniques, succède un dialogue riche de ce que les sujets n'ont pas dit mais auraient pu dire ou exprimer, des thèmes, c'est à dire, au sens de Bakhtine, des formes concrètes d'énonciation intégrant le jugement sur l'objet de l'action et son destinataire.

Ce cadre adopté en formation (initiale ou continue) des enseignants amène ainsi ces derniers, accompagnés par le chercheur, à mettre - ou remettre - au travail des questions telles que l'organisation de leur travail et celui de leurs élèves, dans des espaces de travail renouvelés (FÉLIX; SAUJAT; COMBES, 2012). II se fonde sur le postulat d'une circulation entre la compréhension et la transformation des situations de travail, l'une et l'autre étant réciproquement le moyen et l'objectif de l'intervention-recherche. En amenant les enseignants à reprendre la main sur leur activité, l'espace de formation peut être considéré comme médiateur entre l'activité passée des uns (dont rendent compte les traces filmées par exemple) et celle à venir (usagers, professeurs débutants et formateurs).

\section{Méthodologie}

Afin d'accompagner les enseignants à répondre à la prescription de mise en place d'une évaluation par compétences en cycle 3, notre démarche a consisté à prendre appui sur leurs préoccupations puis sur le partage et la discussion des ressources didactiques ou organisationnelles qu'ils ont eux-mêmes créées. L'intervention-recherche vise dès lors à identifier les savoirs mobilisés, les outils et dispositifs co-élaborés par les différents enseignants et à comprendre en quoi

4 Dont nous détaillerons les étapes dans la présentation de la méthodologie de recherche. 
leur partage peut participer du développement professionnel de chacun et de celui du milieu de travail collectif.

\section{1 Contexte de l'intervention-recherche}

L'intervention-recherche porte sur la mise en œuvre de l'évaluation par compétences, dont l'objectif est de se focaliser davantage sur les processus d'apprentissage et la mobilisation de savoirs face à des situations nouvelles (CLERC, 2012) que sur les performances scolaires. Cette conception de l'évaluation vise à favoriser l'ajustement des enseignements de manière dynamique en fonction des difficultés de chaque élève. Elle insiste sur la convergence entre les situations d'apprentissage et les situations d'évaluation (DROYER; JORRO, 2019 ; MOTTIER LOPEZ; FIGARI, 2012). Le contexte scolaire étudié se particularise par le fait qu'il concerne le cycle 3, à la charnière entre le premier degré (école) et le second degré (collège), au sein d'un réseau d'éducation prioritaire renforcé ${ }^{5}$ qui accueille des élèves de milieu difficile.

Dans ce contexte, l'intervention-recherche regroupe des enseignants volontaires expérimentés ${ }^{6}$ entre sept et 14 ans d'ancienneté dans le métier appartenant à des institutions et des cultures professionnelles différentes avec lesquels il a donc fallu envisager des espaces-temps compatibles selon les disponibiltés de chacun. De fait, nous avons constitué deux sites d'intervention7: a) un site concernant six enseignants du second degré intervenant dans des classes de sixième dans différentes disciplines scolaires, regroupés eux-mêmes en binômes associant respectivement deux collègues en français (Hélène et Élodie), deux en histoire-géographie (Lily et Marc), un en mathématiques (Jean) et une en arts plastiques (Mina); et b) un site concernant deux professeures de la même école, intervenant sur des doubles niveaux CM1-CM2 (Sophie et Anne).

5 Ces réseaux se veulent une réponse à l'échec scolaire grâce notamment à l'instauration d'une continuité du parcours des élèves dans un territoire géographique considéré comme défavorisé.

6 Tous ont entre sept et dix ans d'ancienneté dans le métier.

7 Un troisième site regroupait l'équipe de direction et d'encadrement dont nous ne restituons pas les résultats dans le cadre de cet article. 


\section{2 Dispositif de recherche : étapes, recueil et traitement des données}

Différentes étapes respectant le cadre méthodologique des auto-confrontations ont guidé la recherche.

Dans un premier temps, des entretiens collectifs ont été conduits, respectivement avec les participants de chacun des deux sites afin de faire émerger leurs préoccupations vis-à-vis de la mise en oeuvre de l'évaluation par compétences. L'analyse thématique réalisée à partir de la retranscription verbatim de ces entretiens a permis de restituer et de mettre en discussion avec les acteurs de chaque site leurs principales préoccupations et axes de réflexion.

Dans un deuxième temps, des observations puis des enregistrements filmés ont été réalisés en classe avec les acteurs de chaque binôme constiué au sein des deux « sites enseignants ». Les séquences filmées, considérées comme des traces de l'activité des enseignants, ont servi de support à des entretiens d'auto-confrontation simples (ACS). Les ACS ont été suivies d'entretiens en auto-confrontation croisées (ACC) ou d'entretiens croisés sur la base de photos de classe (en fonction du choix des enseignants) destinés à confronter les manières spécifiques dont les enseignants conçoivent et conduisent les dispositifs d'évaluation par compétences. L'activité discursive des acteurs au cours des ACS et des ACC a été analysée d'un double point de vue : celui des thématiques en lien avec les objets du dialogue et celui des marqueurs langagiers, comme le temps employé, le pronom, les adverbes, etc., qui traduisent l'engagement subjectif et collectif des acteurs au travail (FAïTA, 2011). L'analyse s'est focalisée sur des extraits significatifs au regard de leur capacité à rendre compte des dispositifs d'évaluation et des dilemmes liés à leur usage.

Dans un troisième temps, nous avons organisé des phases de " retour au collectif » (CLOT; FAÏTA 2000) regroupant l'ensemble des binômes des deux sites destinés à mettre en discussion les manières individuelles de mobiliser les outils d'évaluation par compétences. La conduite des ces entretiens, prenant appui sur des extraits filmés ou des photos retenus par les enseignants, visait à favoriser d'éventuels déplacements de chacun ainsi que l'élaboration de ressources communes. L'analyse de ces échanges a permis de prolonger selon les mêmes orientations celles réalisées à l'étape précédente.

Dans un dernier temps, les axes de travail ayant émergé de ces séances ont fait l'objet d'une journée de travail réunissant l'ensemble des acteurs du réseau. 


\section{Résultats}

Les principaux résultats exposés ont trait au choix des dispositifs/outils d'évaluation et aux modalités afférentes de construction du milieu didactique ainsi qu'aux dilemmes de métier mis au travail dans le cadre dialogique de notre méthodologie. Leur présentation prend appui sur des extraits significatifs pointant le rôle de l'activité langagière dans le questionnement des acteurs et la co-élaboration de pistes de transformation de leur activité d'évaluation.

\subsection{De la formulation des préoccupations initiales à l'émergence d'objets de tra- vail}

L'analyse des verbatim des différents entretiens montre la focalisation des enseignants sur les outils et dispositifs d'évaluation qu'ils mettent en place. La récurrence des énoncés relatifs au choix des outils traduit une focalisation sur les modalités de conception et de conduite du travail en classe qui interroge le repérage et le choix des multiples ressources existantes ainsi que les conditions de leur prise en main dans le contexte de la classe.

La centration sur le choix des outils s'avère particulièrement présente chez les deux enseignantes du premier degré, Anne et Sophie. Dans leurs séances, ces enseignantes organisent l'espace et le temps de la classe autour d'une multitude de dispositifs, comme le "classeur d'autonomie" ou le "plan de travail", la classe "Dojo" ou le "tableau de classe" soutenus par différentes modalités de travail le plus souvent individualisées, par exemple l'auto évaluation, le tutorat, l'auto-correction ou les échanges entre élèves. Ces modalités de travail s'inspirent des diverses ressources issues de leur formation initiale et de sites internet, notamment celui du ministère de l'éducation nationale (EDUSCOL) ou de collègues. Elles ont également trait aux diverses méthodes d'apprentissage, par exemple la "grammaire Picot" qui repose sur des textes extraits de la littérature de jeunesse, la " méthode Peroz " qui propose un protocole pour évaluer l'oral et enrichir la prise de parole des élèves ou la "méthode heuristique" de Nicolas Pinel en mathématiques, qui est en fait une synthèse de toutes les pratiques enseignantes.

Dans les entretiens, Anne et Sophie évoquent le caractère chronophage et peu confortable de cette superposition d'outils ainsi que leur difficulté à s'emparer des diverses ressources trouvées sur internet, comme en témoigne l'énoncé suivant extrait de l'ACS avec Anne: C'est difficile, le temps de comprendre l'appli- 
cation, comment ça fonctionne; Je farfouille partout, et là où je trouve quelque chose d'intéressant je l'extirpe, je le télécharge et j'adapte.

Sans entrer dans le détail d'une analyse langagière, on peut remarquer quelques façons de dire singulières, notamment chez Anne, qui pour expliquer son dispositif de "classeur d'autonomie", prend la parole à la place de l'élève à plusieurs reprises: Quand il en arrive à l'intitulé de la fiche, "je peux prendre un travail autonome" ; S'ils ont envie de se rattraper sur le contrôle d'avant, ils peuvent dire: "si je me sens prêt, c'est bon, ... ". Ces changements de postures professeur-élèves dans son discours semblent indiquer qu'Anne est sans arrêt soucieuse de la distribution des rôles qu'autorisent les dispositifs complexes qu'elle met en place, alors que du côté de Sophie cette distribution est plus simple, fluide et clairement établie. Anne est telle un metteur en scène qui veut prévoir le moindre détail et accessoire de la représentation, sans forcément toujours penser au sens de la pièce de théâtre. Remarquons que lors de l'explication de Sophie au sujet de "son plan de travail" à nouveau Anne s'exprime comme si elle était une élève (quitte à ce que moi je fasse l'activité), ce qui semble l'aider à incorporer les explications de Sophie. Anne constate que non seulement les visées d'autonomie de sa collègue sont plus finement pensées, mais que son organisation de classe est plus pertinente : C'est déjà un truc en plus par rapport à moi.

Cette façon de parler, sortant les expressions linguistiques d'un contexte d'usage communicationnel, aide Anne à mieux comprendre les explications de sa collègue qui deviennent des objets de réflexion et d'analyse. Elle adopte un langage social, une façon de parler caractéristique d'un groupe particulier (les élèves) dans une situation socioculturelle particulière (la classe). Selon les thèses Bakhtiniennes, le mot au sein du langage est à moitié celui d'un autre. Il devient le « sien propre » à condition que l'opérateur le fasse vivre dans sa propre intention. Notons que Sophie ne relève pas ce changement d'identité ; les paroles d'Anne font partie d'un énoncé quotidien pourvu de significations sous-entendues par les deux protagonistes qui partagent un horizon spatial commun, ainsi que la connaissance, la compréhension, et l'évaluation de la situation.

Dans l'ACC, face à la diversité des outils et ressources qu'elles énoncent, les deux enseignantes reviennent sur les manières dont elles tentent d'adapter les outils en lien avec leur préoccupation d'impliquer les élèves dans leurs apprentissages. Elles évoquent ainsi un autre dispositif, celui "des ceintures" également utilisé au collège qui se réfère aux arts martiaux : l'élève demande au professeur de "passer la ceinture"quand il pense maîtriser le niveau requis d'une compétence : 
Ch : Et cette mise en place des ceintures pour toi ça sert à quoi ?

Anne: Ça les motive à obtenir de plus en plus de droits. Parce que plus je peux leur faire confiance et plus ils ont de libertés dans la classe....

Ch : Et ces droits et devoirs des ceintures c'est toi qui les as créés ?

Anne : Oui. Il n'y a personne qui l'a celui-là. Je me suis basée sur ce qu'avait fait ma tutrice et du coup je l'ai réaménagé en fonction de ce que moi je me sentais capable de faire.

[...]

Sophie : Oui, au départ je m'en servais plutôt pour le côté cahier de vie [...]

Ch: Mais c'est toi-même qui es allée chercher cet outil ?

Sophie : Système de comportement, oui. Je n'étais pas du tout satisfaite d'aucun système de comportement et du coup j'ai trouvé ce système-là en cherchant. Je voulais quelque-chose qui ait du sens et pour les enfants et pour moi.

Ces modalités d'adaptation révèlent des dilemmes entre l'usage des outils, leur conformité aux programmes scolaires et le sens qu'elles accordent à l'évaluation par compétences: Je prends des ressources sur internet mais plus ça va plus je m'en détache parce que j'ai vraiment besoin de me remettre en continuité avec les programmes (Sophie).

Ainsi, les outils et dispositifs d'évaluation mis en place deviennent objet central du dialogue et source de discussion avec son propre métier et celui des pairs, comme le révèle la récurrence de leur évocation au fil des différents entretiens. L'évolution que traduisent les énoncés dans les ACS puis les ACC (ou entretiens à partir de photos) montre le recyclage des pré-occupations initiales des enseignants exprimées lors de l'entretien collectif initial en occupations dans l'action (au sens de Saujat, 2010). Si les enseignantes s'accordent sur plusieurs des outils ou ressources qu'elles mobilisent, certains points de divergence quant à leur conception didactique et à leur usage émergent au cours de l'ACC. Ainsi, en interrogeant leurs propres pratiques, grâce à l'activité dialogique au cours des entretiens d'auto-confrontations, elles énoncent leurs difficultés et s'engagent dans des controverses quant à leurs manières respectives de faire, ce qui provoquera un renouvellement de leurs manières d'utiliser les outils.

\section{2 Des outils d'évaluation mis en place au questionnement de leur usage et de leurs fonctions}

L'évolution des énoncés des enseignants relatifs au choix des outils traduit un déplacement de leur simple description lors des ACS à une réflexion, lors des phases ultérieures de la recherche (ACC et retour au collectif), sur leur usage 
et leurs fonctions du point de vue des apprentissages des élèves.

Par exemple, dans la continuité des échanges relatifs à la mobilisation des outils (voir section 3.1), Anne et Sophie reviennent sur "la fiche nature des mots" qui devrait permettre d'évaluer les compétences des élèves en matière de compréhension grammaticale. Or on comprend qu'Anne effectue un glissement dans l'usage de cette fiche entre logique d'apprentissage et maintien de l'ordre dans la classe: En fonction de leur attitude, de leur comportement, ils peuvent gagner des plus ou avoir des moins. Sophie manifeste son désaccord avec les façons de faire de sa collègue à plusieurs reprises (Non, pas du tout), et rappelle que les quatre règles de la "classe Dojo" qu'elle utilise empêchent la confusion entre évaluation des apprentissages et du comportement.

De même, les échanges des deux enseignants d'histoire-géographie du second degré, Marc et Lily, au cours de l'ACC mettent en évidence les modalités différentielles de mise en place du "dispositif en îlots" et les controverses qui en découlent. Dans les séances filmées, qui portent la découverte de la civilisation romaine autour d'une démarche de problématisation telle que prescrite par les programmes du cycle 3 en 6e, Marc et Lily mettent en place un dispositif en îlot bonifié qui consiste à faire travailler les élèves en groupes en leur donnant la possibilité permanente de gagner (ou de perdre) des "points bonus" pour valoriser leur équipe (mais aussi augmenter leurs points individuels). Des documents écrits (photos, textes) destinés à soutenir le "processus d'enquête" et le prélèvement d'indices documentaires sont proposés aux élèves qui doivent en faire une restitution sous forme de carte mentale (pratiques développées en formation continue disciplinaire). Malgré un dispositif et des objectifs partagés relatifs aux compétences transversales "coopérer-mutualiser" et "s'auto-évaluer", les façons dont ils conduisent la séance diffèrent. Alors que Marc s'appuie sur des documents identiques pour chacun des îlots, Lily propose des documents dont les thématiques et les niveaux de difficulté varient selon les îlots. Ces choix entraînent des stratégies d'intervention spécifiques pour chacun des enseignants: si Marc circule de groupe en groupe pour guider les élèves avant de proposer un temps d'institutionnalisation ${ }^{8}$ grâce au tableau interactif, Lily n'hésite pas à interrompre la classe pour proposer régulièrement des temps d'institutionnalisation prenant appui sur les difficultés qu'elle repère chez les élèves lors des régulations par groupes. Ces différences inhérentes à l'usage de ce dispositif feront l'objet de controverses durant I'ACC à propos de la constitution des groupes et des modalités de différenciation: 
Marc: Oui, les groupes ont changé, ils ont le droit de changer au bout de 20 points verts. Quand ils arrivent à 20 points verts, ils ont la possibilité de modifier leur groupe chaque fois

Lily: Et moi, ils sont plus en îlots bonifiés en fait. J'avais commencé en îlots bonifiés en début d'année, j'en suis sortie, je suis passée à la narration de compétences en fait. Et euh, du coup ils se mettent comme ils veulent du moment que dans la séquence on a une unité.

[...]

Marc: Je pense que la mutualisation des connaissances et des compétences est très utile pour chacun des élèves qu'ils soient plus ou moins en difficulté, euh. Puis ils seront confrontés à ça de toute manière, dans l'avenir aussi,..., et dans la vie, donc euh

Lily : Moi, je suis persuadée qu'en groupes on construit des apprentissages individuels mais il faut très régulièrement le rappeler aux élèves.

Les divergences mises en discussion amènent alors Lily et Marc à remettre en question les fondements mêmes des îlots bonifiés et à questionner les conditions d'une véritable appropriation des compétences:

Lily : Alors, tu vois, moi c'est pour ça que je suis sortie des îlots bonifiés en fait, parce que je trouve que ça induit une compétition qui au final est contraire à l'esprit de coopération qu'on veut insuffler dans la classe. [...] Parce que dans les illots bonifiés t'es pas dans une entrée par compétences, c'est juste une entrée par points en fait, le score. Et du coup, là ça permettait d'entrer complètement dans les compétences et de se faire s'approprier ce coopérer-mutualiser.

[...]

Marc : Ouais, parce qu'on met pas les mêmes termes dessus en fait, mais c'est vrai que j'ai évolué aussi là-dessus, c'est-à-dire que je veux pas que y'ait l'esprit de compétition, ça sert à rien!

Ces échanges au cours desquels chaque enseignant clarifie ses manières de faire tout en dialogant avec son collègue les conduisent à questionner les pistes de transformation possibles en élargissant notamment l'usage des outils à l'ensemble de l'équipe enseignante, ce que permettra le retour au collectif'. Relativement aux indicateurs langagiers, ces interactions se caractérisent par des reformulations, des reprises ou des recontextualisations qui aboutissent à une forme de stabilisation des énoncés traduisant le passage de désaccords à une co-énonciation (au sens de RABATEL, 2012). On voit combien la discussion se nourrit des ressemblances et des dissemblances (WALLON, 1970) que traduisent l'usage des pronoms "je", "tu" et "on", ce dernier marquant une sorte de consensus. L'analyse réalisée par les deux enseignants révèle aussi le poids de l'expérience passée et

9 Comme le montrera l'exposé des résultats dans la section suivante. 
sa remise au travail dans le temps présent et à venir, ce dont témoignent l'usage des temps passé, présent et conditionnel : elle s'enracine dans une histoire, pour partie partagée, et dans un dialogue entre le style et le genre du métier, ancrés dans la culture disciplinaire (CLOT; FAÏTA, 2000).

Au fil des ACS et ACC, les enseignants, appelés à discuter avec leurs pairs de leur travail, reviennent sur le sens de leur activité puis élaborent ensemble des moyens de dépasser les tensions qui les animent à propos des outils mobilisés. L'activité réelle d'apprentissage des élèves représente alors un instrument important de leur développement professionnel lorsque les enseignants, au moyen de méthodes indirectes, la redécouvrent et l'interrogent collectivement à partir de leur propre activité de conception et de conduite des séances en classe (RUELLAND-ROGER ; CLOT, 2013).

\section{3 De cultures de métier différenciées à l'élaboration d'un cadre commun}

Les débats professionnels développés lors du retour au collectif regroupant les sites $1^{\text {er }}$ et $2^{\text {d }}$ degrés, montrent les difficultés que rencontrent les acteurs à mettre en cohérence les cultures inhérentes à l'école primaire et au collège.

Le projet de constitution d'une "classe de 6 e sans note » porté par une équipe de professeurs volontaires du collège amène ainsi les enseignants à prendre conscience des difficultés inhérentes à la cohérence de l'enseignement au sein du réseau, que celle-ci concerne les enjeux organisationnels, pédagogiques, éthiques ou didactiques. Pour les professeurs, la continuité des enseignements se heurte à des problèmes d'interprétation et d'opérationnalisation des prescriptions, ce qui les amène à réfléchir collectivement aux moyens d'harmoniser les enseignements en $6^{e}$ tout en tenant compte des différences de pratiques et cultures scolaires liées aux écoles dont proviennent les élèves (BRIÈRE ; ESPINASSY, 2021).

Lors des réflexions collectives au sujet de la mise en œuvre la " $\sigma^{\text {e sans }}$ note ", Jean (mathématiques) souligne des différences en termes d'apprentissages scolaires selon les écoles primaires fréquentées par les élèves accueillis en $6^{e}$ : Ils viennent tous d'écoles primaires différentes, et ils ont tous des méthodes différentes. Ces différences vont prendre corps pour ces enseignants lorsqu'ils évoquent ensemble le Livret Scolaire Unique qui suit l'élève du début de l'école primaire à la fin du collège - cycles 2, 3 et 4: ces éléments sont numérisés dans une application informatique nationale dont l'enjeu est justement d'établir une continuité pédagogique dans la scolarité de l'élève. Les enseignants remarquent 
pour le cycle 3 qui les concerne tous, que la validation des compétences effectuée par les enseignants de l'école primaire en fin de CM1 et CM2 (appelé "tableau d'apprentissages "selon Sophie) ne parviennent pas jusqu'aux professeurs du collège qui à leur tour évaluent ces mêmes compétences transversales en fin de $6^{e}$. Ce manque de cohérence manifeste dans la conception et l'usage des outils imposés par l'institution sera source de débat et de questionnement sur la définition partagée des critères d'évaluation, parfois sur un mode ironique: Existe-t-il une compétence "avoir la bonne réponse" (Lily) ? Quels sont les critères de la compétence "avoir de l'imagination " (Sophie) ? Le même type de problème est évoqué par les professeurs du collège au sujet du bulletin scolaire de mi-trimestre qu'ils doivent concevoir pour la " 6 e sans note " afin de le communiquer aux élèves et leurs parents. Habituellement au collège, c'est l'application "Pronote"qui est utilisée et pour laquelle les professeurs n'indiquent pas de notes mais des couleurs comme l'explique Mina: Je ne rentre plus que la couleur car sur Pronote l'évaluation par compétences se fait par code-couleur.

La question de la cohérence des codes-couleur remet en débat le " dispositif des ceintures" : les acteurs en présence avouent leur ignorance non seulement à propos de la continuité entre les couleurs de ceinture utilisées au primaire et au collège, mais davantage encore entre les codes-couleur des ceintures et de Pronote. Lily précise que :

Les couleurs, de toute façon, d'une classe à l'autre, elles ne sont pas les mêmes. Entre les ceintures de comportement, les ceintures de conjugaison, les ceintures de mathématiques, les ceintures de calcul mental, ... Ce qui est important, c'est de donner à un moment donné, aux élèves, et aux parents, la signification.

Nous comprenons que malgré la mobilisation par tous les enseignants du "dispositif des ceintures", les manières de faire et les logiques qui les soustendent divergent fortement d'une enseignante à l'autre, allant à l'encontre de la continuité pédagogique pourtant recherchée. La coordonnatrice du réseau, issue du premier degré, fait part de son expérience de parent d'élève : C'est vraiment important cette histoire de couleurs. Moi j'ai eu un quiproquo avec ma fille parce qu'elle est revenue du collège avec du bleu en comportement mais en fait, c'était mieux que le vert, affirmation que partage Lily, mais pas la coordonnatrice : Bah non, pour moi, ce n'était pas comme ça... Et je me suis fâchée contre elle! Tous parviennent à rire de cet épisode malgré le désarroi qu'il provoque dans le 
groupe. Bakhtine considère le rire comme affranchissement des dissonances ou des conflits d'une activité : s'il prend part au processus d'émancipation symbolique des tensions réelles, comme puissance active dans la situation, alors, il faut regarder le rire comme un geste du travail efficace (BAKHTINE, 1984).

Pour résumer, le cadre dialogique instauré par notre intervention a suscité des échanges entre ces enseignants qui se heurtent à diverses difficultés. Ces discussions mettent en lumière plusieurs types de déplacements individuels et collectifs opérés par les acteurs. À titre d'exemples individuels: Anne prend conscience de certaines de ses rigidités d'usage d'outils qu'elle détourne par ailleurs de leur fonction évaluative ; Sophie est ébranlée par le constat de l'accumulation peu productive des dispositifs dans sa classe ; Lily s'aperçoit que la ritualisation obsessionnelle des cartes mentales prive parfois les élèves d'autonomie, etc. Mais collectivement, en ce qui concerne le cycle 3 qui les réunit, les enseigants parviennent d'une part à identifier les obstacles organisationnels qui les empêchent de travailler ensemble, et d'autre part, à nommer des principes didactiques et pédagogiques partagés. Ces dialogues fructueux leur permettent d'envisager ensemble de nouvelles perspectives visant la continuité des apprentissages de leurs élèves de l'école au collège.

\section{Conclusion}

Les résultats de la recherche collaborative présentée montrent le lien étroit entre les modalités de la recherche et ceux de la formation. L'intervention-recherche, en s'immisçant dans le milieu de travail, a pour but de le mettre en mouvement en termes de partage d'expériences, de développement des situations et des acteurs où le langage joue un rôle primordial dans le dialogue instauré avec son propre métier et celui de ses pairs.

Les rencontres organisées par sites, les autoconfrontations par binômes, les regroupements collectifs ont tous pour objectif la prise de conscience du réel de leur activité par les acteurs en présence afin de susciter de nouvelles manières de faire son travail. "La conscience n'existe pas comme un état mental séparé mais comme un rapport réel [...] C'est seulement en mouvement qu'elle montre ce qu'elle est " (CLOT, 2003, p. 11). Le milieu de travail est un milieu particulièrement propice au développement car il occasionne sans cesse des discordances entre ses actions (motivées par l'atteinte d'un but immédiat) et le sens qu'un sujet donne à son activité, comme en attestent les pratiques d'Anne détour- 
nant certains dispositifs d'évaluation de savoirs pour les transformer en outils de contrôle du comportement des élèves. Le milieu de recherche et d'action que nous avons créé lors de notre intervention représente une zone commune de développement pour des compétences hétérogènes se spécifiant mutuellement (CLOT, 1995, p. 176). C'est particulièrement manifeste lorsque des cultures différentes se rencontrent comme celles des premier et second degrés dans les résultats présentés ici. Cette zone a ses règles, fondées sur ce que Bakhtine nomme un "paradigme exotopique " qui, dans le domaine de la culture, est le moteur le plus puissant de la compréhension: "Une culture étrangère ne se révèle dans sa complétude et dans sa profondeur qu'au regard d'une autre culture " (BAKHTINE, 1984, p. 347-348). Dans les interventions-recherches, la construction de ces milieux sociaux d'analyse et d'actions comme instruments de recherche permet d'être au cœur des situations et de les faire dialoguer avec d'autres tout en maintenant la dynamique d'échanges intersubjectifs qui met en mouvement l'activité existante. Ces échanges dialogiques deviennent alors un instrument de transformation de l'expérience en inscrivant "les traces de ces changements dans une histoire personnelle possédant son propre cours, distinct de tel ou tel échange, fait à la fois de potentialités et d'opérationnalités encore inaccomplies " (CLOT, 2000, p. 146). En ce sens, on peut considérer que les professionnels engagés dans les recherches collaboratives s'engagent également dans un processus de formation continue.

Par ailleurs, les situations réelles de travail peuvent être exploitées dans les dispositifs de formation initiale des futurs enseignants selon deux modalités complémentaires : celle d'objet à comprendre afin de le transformer et celle d'instrument de formation soumis à l'analyse (ESPINASSY, 2016) pour leur permettre de circuler entre expériences pratiques (situations de stages), formation théorique à/par la recherche et analyse réflexive afin de favoriser l'acquisition de compétences professionnelles (ESPINASSY; BRIÈRE-GUENOUN ; FÉLIX, 2018). L'espace de la formation devient celui du travail partagé, offrant aux formés la possibilité de convertir les difficultés vécues sur un mode personnel en un questionnement de métier partagé, où l'ordre dialogique permet aux sujets de se rencontrer, euxmêmes et les autres, en lien avec leurs histoires, leurs environnements et les circonstances. Ce qui nous intéresse dans cette conception des rapports langagiers de l'individu à son milieu de travail, ce sont bien les compromis, les hésitations, les recherches de mots qui témoignent de la réalisation de la pensée et de la prise de distance qui s'opère sources du développement du pouvoir d'agir. La for- 
mation peut être pensée comme un milieu riche, déclenchant des mécanismes d'interprétations reciproques, s'adressant au métier comme un " surdestinataire " au sens de Bakhtine (1984).

Finalement, le rapport dialogique instauré en formation s'enracine dans la polyphonie du métier enseignant où différentes voix co-existent et interagissent dans le discours (ESPINASSY, 2016), et où la dimension développementale du langage ouvre la voie aux perspectives compréhensives et transformatives sous-tendues par les actions de recherche et de formation.

\section{Références}

AMIGUES, R. Le travail enseignant : prescriptions et dimensions collectives de l'activité. Les Sciences de l'éducation pour l'Ère nouvelle, 42 (2), p. 11-24, 2009.

BALSLEV, K. ; TOMINSKA, E. ; VANHULLE, S. Le jour où je serai dans ma classe ce sera différent. Entretiens de stage et construction de savoirs professionnels. Les Sciences de l'éducation Pour l'Ėre nouvelle, 44(2), p. 85-101, 2011.

BAKHTINE, M. Esthétique de la création verbale. Paris : Gallimard, 1984.

BRIĖRE, F.; ESPINASSY, L. De l'analyse de l'activité aux analyses didactiques: une recherche participative. Mise en œuvre de l'évaluation par compétences en cycle 3 en réseau d'éducation prioritaire. Phronesis, 10 (1). Éditions Champ social, Université de Sherbrooke, Canada, p.18-36, 2021.

BRIÈRE-GUENOUN, F.; ESPINASSY, L. L'évaluation par compétence au sein du réseau REP+ Miramas. Rapport de recherche, Aix-en-Provence, DAFIP-SFERE, 2019.

BRIÈRE, F. ; SIMONET, P. Développement d'étudiants stagiaires en EPS et activité du formateur-chercheur au prisme d'une analyse croisant une approche didactiqe et clinique de l'activité. Education \& didactique, 15 (1), p. 47-74, 2021.

BLÖMEKE, S. ; GUSTAFSSON, J. ; SHAVELSON, R. « Beyond dichotomies », Zeitschrift für Psychologie, vol. 223/1, p. 3-13, 2015. http://dx.doi.org/10.1027/2151-2604/a000194.

BRUNER, J. Culture et modes de pensée. Paris : Retz, 2000.

CLERC, F. Une avancée ambiguë. In VILLENEUVE, J.-L. (Dir.), Le socle commun en France et ailleurs. Paris : Le Manuscrit, 2012.

CLOT, Y. Le travail sans l'homme ? Pour une psychologie des milieux de travail et de vie. Paris: La découverte, 1995.

CLOT, Y. La formation par l'analyse du travail : pour une troisième voie. In MAGGI, B. (Dir.)., Manières de penser, manières d'agir en éducation et en formation, $\S 5, p .133-156$. Paris: PUF, 2000.

CLOT, Y. Vygotski, la conscience comme liaison, In VYGOTSKI, L. Conscience, inconscience, émotions, Paris : La Dispute, p. 7-59, 2003. 
CLOT, Y. Travail et pouvoir d'agir. Paris : PUF, 2008a.

CLOT, Y. La recherche fondamentale de terrain : une troisième voie. Éducation permanente, 177, p. $67-78,2008 \mathrm{~b}$.

CLOT, Y. ; FAïTA, D. Genre et style en analyse du travail. Travailler, n. 4, p. 7-43, 2000.

COFFEY, $\mathrm{H}$. They taught me: The benefits of early community- based field experiences in teacher education. Teaching and Teacher Education, 26 (2), p. 335-342, 2010.

DROYER, N.; JORRO, A. L'évaluation : levier pour l'enseignement et la formation. In : JORRO, A., DROYER, N (Dir.). L'évaluation, levier pour l'enseignement et la formation. Louvain-la-Neuve, Belgique : De Boeck Supérieur, p. 7-14, 2019.

ESPINASSY, L. Une structure dialogique et plastique : une hypothèse de l'usage de Néopass@ ction. In BOBILLIER CHAUMON, M. ; CLOT, Y. (Dir.). Clinique de l'usage : les artefacts technologiques comme instrument de développement de l'activité. Activités [En ligne],p.13-2 | 2016. http://activites.revues.org/2863

ESPINASSY, L. ; BRIÈRE-GUENOUN, F. ; FÉLIX, C. De l'intervention-recherche dans les dispositifs de lutte contre les inégalités scolaires à la formation des enseignants. Recherche et Formation, 87, p.47-60, 2018.

FAIITA, D. Théorie de l'activité langagière. In MAGGI, B. (Dir.) Interpréter l'agir. Un défi théorique. Paris : PUF, p. 41-67, 2011.

FAIITA, D. ; SAUJAT, F. Développer l'activité des enseignants pour comprendre et transformer leur travail : un cadre théorique et méthodologique. In : Yvon, F. ; SAUSSEZ, F. Analyser l'activité enseignante. Des outils méthodologiques et théoriques pour l'intervention et la formation. Laval : Presses de l'Université de Laval, 2010. p. 41-71.

FÉLIX, C. ; SAUJAT, F. ; COMBES, C. Des élèves en difficulté aux dispositifs d'aide : une nouvelle organisation du travail enseignant ? Recherches en Education, HS n4, p. 19-30, 2012.

GLAZER, C. ; ABBOTT, L. ; HARRIS, J. A teacher-developed process for collaborative professional reflection, Reflective Practice, 5(1), p. 33-46, 2004.

HAYMORE SANDHOLTZ, J. Inservice training or professional development: contrasting opportunities in a school/university partnership, Teaching and Teacher Education, 18 (7), p. 815-830, 2002.

LEONTIEV, A.N. Activité, conscience, personnalité, Moscou : Editions du Progrès, 1984.

LESSARD, C. Evaluation des effets du développement professionnel des acteurs de l'éducation. Paris : Cnesco, 2021.

MALET, R. (coord.). De la formation continue au développement professionnel des personnels d'enseignement et d'encadrement éducatif. Situation nationale, comparaisons internationales, état des recherches. Paris : Cnesco, 2021.

MAUBANT, P. ; ROQUET, P. Les reliances de l'alternance en formation et ses effets sur les processus de construction identitaire des alternants. Phronesis, 1 (5), p. 1-3, 2016.

MAYEN, P. Intégrer les savoirs à l'action. In PERRENOUD, P. ; ALTET, M. ; LESSARD, C. ; PAQUAY, L. Conflits de savoirs en formation des enseignants. Entre savoirs issus de la recherche et savoirs 
issus de l'expérience. Bruxelles : De Boeck Université, 2008, p. 43-58.

MOTTIER LOPEZ, L. ; FIGARI, G. (Dir.). Modélisation de l'évaluation en éducation. Questionnements épistémologiques. Bruxelles : De Boeck, 2012.

RABATEL, A. Positions, positionnements et postures de l'énonciateur. In TRANEL, Travaux neuchâtelois de Linguistique, Institut des sciences du langage et de la communication, 56, Neuchâtel, Suisse, p. 23-42, 2012.

RUELLAND-ROGER, D. ; CLOT, Y. L'activité réelle de l'élève : pour développer l'activité enseignante. Revue internationale du CRIRES, innover dans la tradition de Vygotsky, vol 1, 1, p. 20-25, 2013.

SAUJAT, F. Travail, formation et développement des professionnels de l'éducation : voies de recherche en sciences de l'éducation. Note de synthèse pour l'Habilitation à Diriger des Recherches. Université de Provence (Aix-Marseille I), 2010.

SAUJAT, F. ; FÉLIX, C. Activité enseignante à l'état naissant et développement du pouvoir d'agir : un prototype clinique. Phronesis, 7(2), p. 4-14, 2018.

SCHWARTZ, Y. Reconnaissances du travail. Pour une approche ergologique, Paris : PUF, 1997. SIMONET, P. J. Bruner. Pourquoi nous racontons-nous des histoires ? L'orientation scolaire et professionnelle [En ligne], 34/2 | 2005.

SIMONET, P. La transmission du geste, une question de développement. Contribution d'un psychologue du travail. In Terrien, P. ; Güsewell, A. ; Vivien, R. (dir.), Didactique de la musique instrumentale : entre tâche et activité. Paris : L'Harmattan, 2019, p. 27-46.

VEILLARD, L. ; COPPE, S. Mobilisation de connaissances antérieures en formation professionnelle par alternance: Perspectives apportées par une approche comparatiste. Éducation \& didactique, 2(2), p. 45-76, 2009.

VINATIER, I. Pour une didactique professionnelle de l'enseignement. Rennes : PUR, collection Paideia, 2009.

VYGOTSKI, L.S. La conscience comme problème dans la psychologie du comportement. Société française, $n^{\circ} 50$, avril-juin, p.35-49, 1994.

VYGOTSKI, L.S. Pensée et langage. Paris : La Dispute, 1997.

WALLON, H. De l'acte à la pensée. Paris, France : Flammarion, 1970.

WANLIN, P.; CRAHAY, M. La pensée des enseignants pendant l'interaction en classe. Une revue de la littérature anglophone. Éducation \& didactique, 6(1), p. 9-46, 2012.

\section{Sobre as autoras}

Fabienne Brière - MCF-HDR, INSPÉ Aix-Marseille Université, FED 4238 SFERE-Provence, UR 4671 ADEF, Marseille, France. fabienne.briere@univ-amu.fr - https://orcid.org/00000002-5221-5471

Laurence Espinassy - MCF-HDR, INSPÉ Aix-Marseille Université, FED 4238 SFERE- 
Provence, UR 4671 ADEF, Marseille, France. laurence.espinassy@univ-amu.fr - https:// orcid.org/0000-0002-7129-7011 